\title{
Referenciação dos cuidados de saúde primários a uma unidade de psiquiatria da infância e da adolescência do Norte de Portugal: uma análise de dois anos
}

Marta Araújo, ${ }^{1}$ Eva Gomes, ${ }^{2}$ Marta Fernandes, ${ }^{2}$ Sandra Borges, ${ }^{3}$ Sara Melo, ${ }^{3}$ Bárbara Romão, ${ }^{3}$ Graça Mendes ${ }^{4}$

\section{RESUMO}

Objectivos: Caracterizar as referenciações efectuadas pelos Cuidados de Saúde Primários (CSP) à consulta da Unidade de Psiquiatria da Infância e da Adolescência (UPIA) do Centro Hospitalar de Vila Nova de Gaia/Espinho (CHVNG/E); avaliar a comunicação entre os dois níveis de cuidados.

Tìpo de Estudo: Observacional descritivo transversal.

Local: UPIA do Serviço de Psiquiatria e Saúde Mental do CHVNG/E.

População: A amostra incluiu todas as referenciações dirigidas à referida unidade provenientes dos CSP, via ALERT P1®, durante os anos de 2010 e 2011.

Métodos: Procedeu-se à análise descritiva das variáveis género, idade, motivo de referenciação, prioridade da referenciação, prioridade atribuída pelo triador, motivo de recusa/devolução do pedido, tempo de espera, comparência do utente e qualidade da carta de referenciação.

Resultados: A análise incluiu 980 referenciações (61,5\% do género masculino). A faixa etária entre os $6-10$ anos foi a mais referenciada (41,4\%). O motivo mais referenciado foi "Sinais/sintomas do comportamento da criança" (31,9\%). De todos os pedidos, $16,9 \%$ foram considerados urgentes pelo médico de família. Destes, 11,6\% foram considerados prioritários pelo triador. De todas as referenciações, $11,3 \%$ foram recusadas/devolvidas, maioritariamente devido a informação insuficiente (36,1\%). Cerca de $90 \%$ das referenciações consideradas prioritárias foram agendadas num prazo $\leq 60$ dias e quase $100 \%$ das restantes referenciações foram marcadas num prazo $\leq 150$ dias. Das 853 primeiras consultas marcadas, 10,5\% das crianças faltaram à consulta e não pediram remarcação da mesma. Cerca de $20 \%$ das referenciações foram consideradas de boa qualidade e cerca de $14 \%$ de má qualidade.

Conclusões: Os resultados sugerem que a articulação e a comunicação entre o médico de família e o psiquiatra da infância e da adolescência são eficazes, embora alguns aspectos possam ser melhorados, nomeadamente a adequação das prioridades atribuídas e a qualidade da informação dos pedidos de consulta.

Palavras-chave: Psiquiatria da Infância e da Adolescência; Cuidados de Saúde Primários; Referenciação.

\section{INTRODUÇÃO}

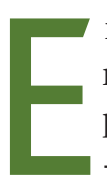

mbora não seja conhecida a prevalência correcta de perturbações emocionais e do comportamento na infância e adolescência, estima-se que 10 a $20 \%$ das crianças tenha um ou mais problemas de saúde mental. ${ }^{1-3}$ Entre os quadros psicopatológicos mais frequentes nestas faixas etárias destacam-se os problemas de comportamento e os pro-

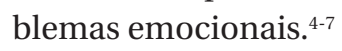

Apesar da prevalência estimada destas patologias, 
apenas uma pequena percentagem destas crianças e jovens entra em contacto com os serviços de saúde mental. ${ }^{4}$ Estima-se que $21 \%$ das crianças apresente uma doença mental ou aditiva e apenas $1 / 3$ a $1 / 5$ destas receba tratamento adequado..$^{1-2,4,8}$

A maioria das situações problemáticas apresenta-se numa fase inicial aos Cuidados de Saúde Primários (CSP). Cabe ao Médico de Família (MF) fazer a avaliação inicial da situação clínica e decidir quanto à necessidade de referenciação aos Cuidados de Saúde Secundários (CSS) tendo em conta as medidas já realizadas, os meios ao seu dispor e o âmbito da sua acção. ${ }^{9}$ Deve ainda utilizar de forma eficiente os recursos de saúde através da coordenação de cuidados com outros profissionais e gerir a interface com outras especialidades. $^{10}$

De forma garantir a continuidade de tratamento, é necessário que os canais de comunicação entre os dois níveis assistenciais funcionem adequadamente, ${ }^{11-16} \mathrm{o}$ que representa uma importante expressão da qualidade da assistência prestada pelo Sistema Nacional de Saúde (SNS)..$^{9,15,17-18}$ e garante um trabalho integrado, mais coeso e eficiente. ${ }^{19}$ Têm sido apontados como possíveis obstáculos a uma boa comunicação a qualidade da informação contida nos documentos de referenciação e o tempo de espera entre o pedido e a marcação da consulta. ${ }^{9,11,13-15,17,20-27}$ A nível nacional têm sido escassos os trabalhos neste campo.

Este trabalho tem como objectivo principal caracterizar as referenciações efectuadas pelos CSP à consulta de Psiquiatria da Infância e da Adolescência (PIA) do Centro Hospitalar de Vila Nova de Gaia/Espinho (CHVNG/E) durante os anos de 2010 e 2011. Como objectivo secundário propomo-nos a avaliar a qualidade da comunicação entre estes dois níveis de cuidados através da aferição da qualidade da carta de referen-

\footnotetext{
Grau, título(s) profissionais e/ou académicos dos autores:

1-2Médica interna de Formação Específica de Medicina Geral e Familiar

${ }^{3}$ Assistente Hospitalar em Psiquiatria da Infância e da Adolescência

${ }^{4}$ Assistente Graduada em Psiquiatria da Infância e da Adolescência e Responsável pela Unidade de Psiquiatria da Infância e da Adolescência do Centro Hospitalar Vila Nova de Gaia/Espinho (CHVNG/E)

Serviço, departamento ou instituição onde trabalham:

'Unidade de Saúde Familiar Arco do Prado - ACeS Grande Porto VII - Gaia

Unidade de Saúde Familiar Nova Via - ACeS Grande Porto VIII - Espinho/Gaia

${ }^{3-4}$ Unidade de Psiquiatria da Infância e da Adolescência do Serviço de Psiquiatria e Saúde Mental do CHVNG/E
}

ciação, o tipo de informação de retorno por parte do médico triador para o MF e a determinação do tempo de espera pela marcação da consulta.

\section{MÉTODOS}

Trata-se de um estudo observacional descritivo transversal que decorreu na Unidade de Psiquiatria da Infância e da Adolescência (UPIA) do Serviço de Psiquiatria e Saúde Mental do CHVNG/E entre Junho e Outubro de 2012, tendo sido obtida autorização do respectivo conselho de administração e o parecer favorável da Comissão de Ética para a Saúde da Administração Regional de Saúde do Norte.

A amostra é um censo constituído por todas as referenciações provenientes dos CSP, via ALERT P1® (sistema electrónico de referenciação dos pedidos de primeira consulta de especialidade hospitalar), à consulta externa da UPIA do CHVNG/E, de 01/01/2010 a $31 / 12 / 2011$. Foram excluídas as referenciações não provenientes dos CSP e as realizadas por outro canal de comunicação que não a plataforma electrónica ALERT P1@.

As variáveis estudadas foram o género, a idade, o motivo de referenciação, a prioridade da referenciação, a prioridade atribuída pelo triador, o motivo de recusa/devolução do pedido, o tempo de espera, a comparência do utente e a qualidade da carta de referenciação.

O motivo de referenciação foi codificado de acordo com a Classificação Internacional de Cuidados Primários, $2^{\text {a }}$ edição (ICPC-2 - International Classification of Primary Care, 2nd edition). ${ }^{28}$ A codificação foi atribuída com base na descrição realizada pelo MF, sempre pelo mesmo elemento da equipa de investigação.

A qualidade da carta de referenciação foi avaliada através da análise do conteúdo desta e classificada como "Boa”, "Aceitável” ou "Má”, de acordo com as recomendações de Irazábal e Gutierres ${ }^{14}$ modificadas de Morera et al $^{12}$ (Quadro I), previamente utilizadas em outros estudos nacionais ${ }^{9,24}$ e internacionais ${ }^{11-12,14,17,20}$ já publicados. A classificação foi atribuída apenas por um elemento da equipa de investigação.

O tratamento estatístico dos dados foi efectuado recorrendo a estatística descritiva, tendo como base a distribuição de frequências e as medidas de tendência central. 


\section{QUADRO I. Níveis de qualidade da referenciação ${ }^{12,14 *}$}

\section{BOA}

Identificação do utente e do médico

Idade do utente, antecedentes pessoais e familiares

Medicação habitual

Dados suficientes sobre a doença actual, exame objectivo e exames complementares

Hipótese diagnóstica, diagnóstico diferencial ou dúvidas a esclarecer

\section{ACEITÁVEL}

O motivo da referenciação está bem claro

Apresenta dados suficientes sobre a doença actual, mas não todos os incluídos na definição de boa qualidade

MÁ

llegível

Sem dados suficientes para a avaliação da doença actual

Ausência de informação

*Irazábal e Gutierres ${ }^{14}$ modificadas de Morera et al. ${ }^{12}$

\section{RESULTADOS}

Durante os anos de 2010 e 2011 houve um total de 980 referenciações, a maioria das quais relativas a crianças do género masculino $(61,5 \%)$. As crianças em idade escolar foram referenciadas com maior frequência $(41,4 \%)$, tendo-se seguido os adolescentes $(29,1 \%)$. O motivo mais referenciado foi "Sinais/sintomas do comportamento da criança" (P22), com 31,9\% de frequência, seguido de "Perturbação hipercinética" (P81) com 12,4\%.

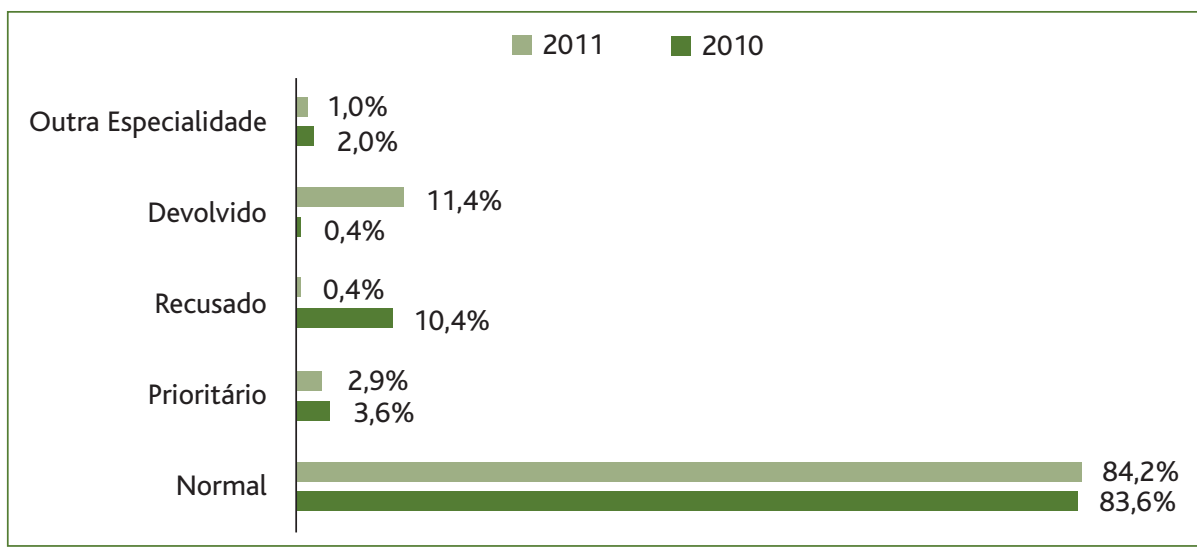

Figura 1. Distribuição dos resultados da triagem, por ano.
QUADRO II. Cruzamento da distribuição da prioridade das referenciações atribuídas pelo Médico de Família com a distribuição da prioridade das referenciações atribuída pelo médico triador

\begin{tabular}{|l|l|l|l|}
\hline \multicolumn{2}{|c|}{} & \multicolumn{2}{c|}{ Pedido } \\
\cline { 3 - 4 } \multicolumn{2}{|c|}{} & $\begin{array}{l}\text { Normal } \\
\mathrm{n}(\%)\end{array}$ & $\begin{array}{l}\text { Urgente } \\
\mathrm{n}(\%)\end{array}$ \\
\hline \multirow{3}{*}{ Triagem } & $\begin{array}{l}\text { Normal } \\
\mathrm{n}(\%)\end{array}$ & $693(97,9 \%)$ & $129(88,4 \%)$ \\
\cline { 2 - 4 } & $\begin{array}{l}\text { Prioritário } \\
\mathrm{n}(\%)\end{array}$ & $15(2,1 \%)$ & $17(11,6 \%)$ \\
\hline & $708(100 \%)$ & $146(100 \%)$ \\
\hline
\end{tabular}

Do total de referenciações, $11,3 \%$ foram recusadas/devolvidas (Figura 1), maioritariamente devido a informação insuficiente $(36,1 \%)$ e a ausência de critérios psicopatológicos $(35,5 \%)$. De todos os pedidos, $16,9 \%$ foram considerados urgentes pelo MF. Destes apenas $11,6 \%$ foram considerados prioritários pelo triador (Quadro II). Em contraste, das referenciações consideradas normais pelo MF 2,1\% foram consideradas prioritárias pelo triador.

A maioria das consultas prioritárias $(90,6 \%)$ foi agendada num prazo $\leq 60$ dias e a quase totalidade das consultas não prioritárias $(99,6 \%)$ foi marcada num prazo $\leq 150$ dias. Das 853 primeiras consultas marcadas, $10,5 \%$ das crianças faltaram à consulta e não pediram remarcação da mesma.

Relativamente à qualidade do conteúdo das cartas de referenciação, 20,4\% das referenciações foram consideradas de boa qualidade, $13,8 \%$ de má qualidade e as restantes - a maioria, totalizando $65,9 \%$ - foram consideradas de qualidade aceitável (Figura 2).

\section{DISCUSSÃO}

Este trabalho incluiu a avaliação de um elevado número de referenciações, constituindo uma amostra bastante superior ao encontrado nos estudos de referenciação nacio- 
nais e internacionais consultados. ${ }^{9,11,14-15,17,20,24-25,29}$ Corresponde também a uma grande área de referenciação (mais de 70 unidades de saúde distribuídas por quatro ACeS diferentes).

Apesar da dimensão da amostra, estes dados não estimam a prevalência de psicopatologia em idade pediátrica, nem em contexto de CSP nem em contexto hospitalar. Reflectem apenas os dados relativos aos utentes que são referenciados para os CSS pelo MF, escapando a esta análise os que são referenciados ao Serviço de Psicologia e Orientação Escolar (SPOE) ou à psicóloga do ACeS, todos os que são acompanhados exclusivamente nos CSP (a maioria), ${ }^{30}$ os provenientes do serviço de urgência, os que são referenciados por outras especialidades hospitalares e por outros hospitais, pela Comissão de Protecção de Crianças e Jovens (CPCJ), pelo tribunal ou serviço social.

No que diz respeito à distribuição por género, os dados evidenciam um claro predomínio do género masculino, o que está de acordo com a bibliografia consultada. ${ }^{4,29}$ No entanto, é importante destacar que esta comparação deve ser valorizada com cautela, uma vez que os estudos nacionais consultados se baseavam numa população ligeiramente diferente (Encarnação et $a l^{4}$ estudou toda a consulta de PIA e Vilela et $\boldsymbol{l}^{29}$ estudou apenas a consulta de primeira infância). De salientar que esta tendência também se verifica quando se avalia a referenciação global em idade pediátrica ${ }^{9}$ (isto é, para todas as especialidades). Diversas hipóteses têm sido apontadas para justificar esta distribuição, nomeadamente uma maior vulnerabilidade genética e fragilidade biológica do género masculino, uma maior capacidade de adaptação sócio-cultural por parte do género feminino ou mesmo uma menor tolerância familiar e social aos sintomas mais disruptivos do género masculino. ${ }^{4,31-34}$ Foi também encontrada uma discreta tendência para o equilíbrio entre os dois géneros na fase da adolescência, também descrita na literatura. ${ }^{4}$

As crianças em idade escolar foram as mais referenciadas (dado concordante com a literatura nacional). ${ }^{4}$ Se analisarmos a distribuição etária da nossa amostra por idades isoladas verifica-se um aumento abrupto de referenciações a partir dos seis anos de idade (que poderá estar relacionado com a entrada no ensino básico) e um pico de frequência aos 10 e aos 12 anos. Daí

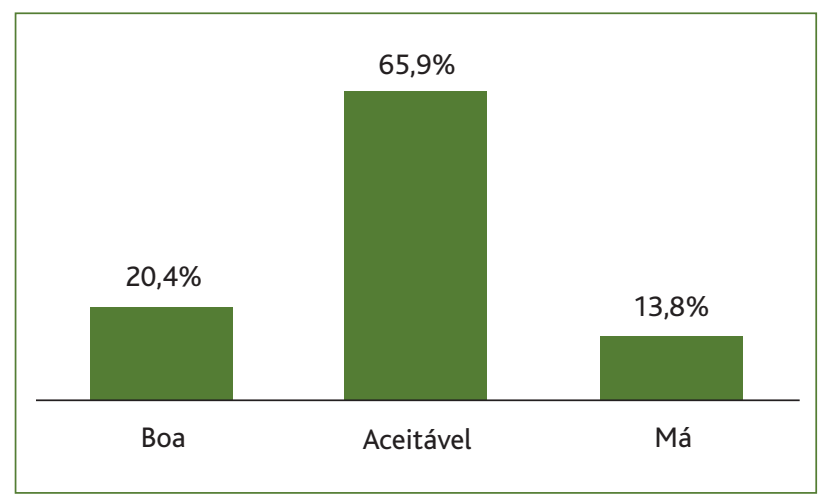

Figura 2. Distribuição da qualidade do conteúdo das cartas de referenciação.

surgir na literatura uma hipótese explicativa que se prende com a possibilidade destas idades constituírem um marco em que as dificuldades de aprendizagem escolar se tornam mais evidentes e que correspondem, simultaneamente, a fases críticas no percurso escolar com exigência crescente. ${ }^{4}$

O principal motivo de referenciação encontrado, à semelhança de outros estudos, ${ }^{4,30-35}$ está relacionado com alterações do comportamento na criança e no adolescente. Mais uma vez, a comparação com a bibliografia referida não deve ser feita directamente, uma vez que os sistemas de classificação utilizados foram diferentes (ICPC-2 no presente estudo versus DSM-IV (Diagnostic and Statistical Manual of Mental Disorders, 4th.Edition) na literatura encontrada) e a população seleccionada também é distinta (enquanto o presente estudo incluiu apenas as referenciações provenientes dos CSP exclusivamente por via informática, os estudos encontrados incluíram todas as referenciações, nomeadamente as provenientes do serviço de urgência, os pedidos de colaboração interna por outras especialidades, as referenciações provenientes das escolas, CPCJ, da psicologia e de outros técnicos, do tribunal, do serviço social, de outro hospital ou pedidos por iniciativa própria). A acrescer aos factos anteriores, é ainda de salientar que os outros estudos encontrados avaliaram não só os motivos de primeiras consultas, mas também os motivos das consultas de seguimento.

O facto de mais de $85 \%$ das referenciações ter sido aceite após o processo de triagem pelo médico psiquiatra demonstra o grau de adequação da referencia- 
ção proveniente dos CSP. Apesar disto, 11,3\% das referenciações foram recusadas ou devolvidas. Só foi encontrado um estudo que avaliava este parâmetro e apresentou um valor bastante inferior ao encontrado $2,1 \%$-, tendo sido os principais motivos de recusa sobreponíveis aos encontrados neste estudo, à excepção dos recusados para avaliação psicológica no ACeS e no (SPOE), o que pode explicar em parte a discrepância dos valores. ${ }^{24}$

Um dado curioso prende-se com a inversão das percentagens relativas à recusa/devolução do ano de 2010 para o ano de 2011, transmitindo a noção de que os pedidos "recusados" em 2010 se convertem em "devolvidos" em 2011. Este dado pode dever-se ao facto de o acto de devolver a referenciação permitir (ao contrário da decisão de recusar) a atribuição de uma justificação que é enviada ao MF (informação de retorno), o que constitui uma mais-valia na comunicação entre os dois níveis de cuidados e que permite uma atitude pedagógica que, em última análise, permitirá a melhoria da qualidade do processo de referenciação.

Na bibliografia consultada, apenas um estudo avaliou a percentagem de pedidos considerados urgentes pelo MF e encontrou um valor de $11,8 \%,{ }^{20} \mathrm{um}$ valor bastante inferior ao encontrado neste estudo (17\%). Um outro dado digno de reflexão é o cruzamento entre a prioridade assinalada pelo MF na carta de referenciação e a prioridade atribuída após pelo psiquiatra triador. Apenas $11,6 \%$ dos pedidos assinalados como urgentes pelo MF foram considerados prioritários pelo triador. Talvez este facto se deva a sobrevalorização por parte dos CSP; subvalorização pelos CSS; dificuldades de comunicação, como a incapacidade de transmitir a gravidade da situação clínica; ou a uma tentativa de manipulação do sistema (para que seja triada mais rapidamente e a consulta tenha um menor tempo de espera). Por outro lado, $2,1 \%$ dos pedidos assinalados como normais foram considerados prioritários após triagem. Estes poderão corresponder a situações subvalorizadas pelos CSP (o que pode estar relacionado com lacunas formativas na identificação de situações clínicas potencialmente graves na área da psicopatologia pediátrica), podem também estar associados a sobrevalorização por parte dos CSS ou, mais uma vez, a dificuldades de comunicação/interpretação da informação dada.
Quanto à análise dos motivos de recusa/devolução detectou-se, exclusivamente em 2011, a referência a devoluções cuja justificação se fazia pela necessidade de avaliação pelo SPOE ou pelo serviço de psicologia do ACeS. Este dado resulta do facto de, a partir do início de 2011, o serviço de PIA do CHVNG/E ter começado a encaminhar determinadas referenciações para os SPOE nos Agrupamentos Escolares, de acordo com as suas áreas de actuação (principalmente nas situações de dificuldades de aprendizagem), bem como da criação de um protocolo que define situações clínicas que devem ser avaliadas inicialmente pela psicologia e, por este motivo, estas situações também passaram a ser devolvidas para serem encaminhadas para o serviço de psicologia do ACeS. Os dados sugerem que o período de 2011 tenha correspondido a uma fase de divulgação destas orientações entre os MF, durante a qual as situações em causa continuaram a ser, inadequadamente, referenciadas à unidade de PIA.

A Portaria no 1529/2008, de 26 de Dezembro, ${ }^{36}$ regula a "Consulta a Tempo e Horas" e determina o tempo máximo recomendado para o agendamento de consultas consideradas normais (< 150 dias) e prioritárias ( $<60$ dias). Encarnação et al,,$^{4} \mathrm{o}$ único estudo nacional encontrado que avaliou o tempo de espera para uma unidade de PIA, não definiu os seus tempos de acordo com a portaria referida mas em função da origem dos pedidos e encontrou tempos de espera médios entre a referenciação e a primeira consulta de 55 dias para os pedidos provenientes do Serviço de Urgência e de 151 dias para os casos sinalizados pela escola. Já Santos $e t$ $a l^{9}$ reportou uma percentagem de $21,5 \%$ das consultas realizadas aos 60 dias e de $71,9 \%$ aos 150 dias, mas não considerou a urgência do pedido, referindo-se à referenciação global em idade pediátrica. Um outro estudo nacional, ${ }^{15}$ este referente à referenciação global em idade adulta, encontrou um tempo de espera médio de 82,5 dias e descreveu a distribuição dos tempos de espera da seguinte forma: $25 \%$ no primeiro mês, $25 \%$ entre um e três meses e $25 \%$ após três meses. Também neste estudo não foi considerada a urgência do pedido. Em 2006, um outro estudo nacional, ${ }^{25}$ também relativo à referenciação global, obteve uma demora média nas consultas de 58,2 dias, tendo metade dos doentes sido atendidos nos primeiros 39 dias. Tendo estes valores como referência, os nossos resultados são bastante satisfató- 
rios em comparação com a bibliografia consultada. ${ }^{4,9,15,25}$

Da pesquisa bibliográfica realizada não foi possível encontrar estudos que permitissem a comparação directa relativamente às faltas/desistências. No entanto, no estudo de Manuel Janeiro ${ }^{15}$ (estudo que analisou referenciação global) foi encontrada referência a esta variável e as faltas/desistências representavam $8 \%$ das consultas marcadas. No artigo de Ponte et $\boldsymbol{l}^{4}$ (que também avalia a referenciação global), a percentagem de consultas não efectuadas é de cerca de $15 \%$ (9\% das quais por falta e as restantes por motivos desconhecidos ou por morte). Seria interessante estudar esta população em particular e averiguar quais os motivos para o absentismo. A área de residência e a distância geográfica desta população já foi apontada como uma das causas. ${ }^{4}$

Não foram encontrados critérios nacionais ou internacionais validados sobre qualidade das cartas de referenciação. O sistema aplicado, apesar de já ter sido usado em vários estudos nacionais e internacionais publicados, 9,11-12,14,17,20,24 está sujeito a algum grau de subjectividade, uma vez que não está sujeito a um sistema de classificação quantitativo. Os resultados obtidos no presente estudo são melhores do que os encontrados em outros estudos que utilizaram os mesmos crité$\operatorname{rios}^{11,17,20,24}$ e em que a percentagem de cartas de boa qualidade variou entre $9,4 \%$ e $20,6 \%$ e as de má qualidade entre $20,5 \%$ e $60 \%$ (excepto quando comparado com o estudo de Irazábal ${ }^{14}$ - que utilizou uma metodologia diferente e que obteve $42,0 \%$ de cartas com boa qualidade e $5 \%$ de má qualidade - ou com o estudo Santos et $a l^{9}$ que teve resultados muito superiores aos da restante bibliografia $-69,6 \%$ de cartas com boa qualidade - e que se refere, ao contrário dos restantes estudos, a toda a população em idade pediátrica, mas que avalia apenas as referenciações de duas Unidades de Saúde Familiar). No entanto, não podemos afirmar que a qualidade das referenciações do nosso estudo seja superior à de outros estudos que avaliaram o mesmo parâmetro, porque é necessário ter em conta que no nosso estudo apenas foram utilizadas referenciações em suporte informático, o que condiciona a ausência de cartas de referenciação ilegíveis (que em alguns estudos mostraram ser responsáveis por cerca de 7 a $10,7 \%$ das referenciações), ${ }^{11,13,24}$ com identificação incompleta/errada ou sem referência à idade e/ou géne- ro, falhas por vezes presentes em cartas de referenciação manuais. Por outro lado, este estudo tem características particulares: por se tratar de uma consulta de pedopsiquiatria deu-se muita importância à referência contexto familiar, dado que não seria tão relevante na referenciação a outras especialidades.

Numa análise final deste estudo destacam-se três pontos fortes: 1) a amostra é de grandes dimensões; 2) os dados foram recolhidos após a primeira consulta hospitalar, permitindo a avaliação da totalidade das referenciações realizadas e impedindo a perda de casos que ainda não tivessem consulta marcada; 3) tanto os MF como os psiquiatras não tinham conhecimento da intenção de avaliação das referenciações, não tendo havido interferência no seu comportamento de referenciação.

Como limitações ao estudo apontamos o facto de a codificação dos motivos de referenciação e da avaliação qualidade da carta de referenciação ter sido atribuída apenas por uma investigadora. Optou-se por esta metodologia numa tentativa de reduzir a subjectividade. No entanto, há vários estudos em que todos os investigadores fazem a avaliação dos pedidos, havendo posteriormente lugar a uma reunião de consenso. Provavelmente teria sido uma melhor opção também para este estudo. Além disto, também a avaliação das cartas de referenciação foi feita apenas de forma subjectiva, não tendo sido avaliada segundo nenhuma escala de pontuação, ou seja, a avaliação foi qualitativa em vez de quantitativa, o que pode constituir uma limitação, principalmente por não ter havido a identificação isolada dos itens em falta na carta de referenciação, o que permitiria uma intervenção dirigida.

Apesar de termos verificado que a maior parte das cartas de referenciação para o serviço de PIA têm qualidade aceitável ou boa e que a maioria dos tempos de espera cumpre os tempos recomendados, há sempre margem para melhorar. A adequação das referenciações e a qualidade das mesmas são dois pontos que podem ser melhorados e com isto os tempos de espera também poderão ser melhorados.

A criação e a distribuição de protocolos de referenciação elaborados pelos serviços de PIA dos CSS com os CSP seriam, sem dúvida, uma mais-valia para melhorar a referenciação a essa especialidade, assim como a existência de consultadorias periódicas nas unidades de saúde de cada ACeS, que também constituem uma 


\section{ferramenta eficiente para colmatar as falhas ainda exis- tentes, uma vez que já foi provado que a realização de reuniões com discussão de casos melhora a qualidade das referenciações. ${ }^{9}$}

\section{AGRADECIMENTOS}

Os autores agradecem ao Professor Doutor Luís Alves pelo apoio e disponibilidade demonstrados durante a realização do presente trabalho.

\section{REFERÊNCIAS BIBLIOGRÁFICAS}

1. Marques C, Torrado M, Natário A, Proença MJ, Coordenação Nacional para a Saúde Mental, Administração Central do Sistema de Saúde. Rede de referenciação hospitalar de psiquiatria da infância e da adolescência. Lisboa: ACSS; s.d. Available from: http://www.acss.min-saude.pt/

2. Marques $C$, Cepêda T. Recomendações para a prática clínica da saúde mental infantil e juvenil nos cuidados de saúde primários. Lisboa: Coordenação Nacional para a Saúde Mental; 2009. ISBN 9789899626324

3. Organização Mundial de Saúde. Relatório mundial da saúde - Saúde mental: nova compreensão, nova esperança. Lisboa: Direcção-Geral da Saúde; 2002.

4. Encarnação R, Moura M, Gomes F, Da Silva PC. Caracterização dos casos observados numa equipa de psiquiatria da infância e adolescência: um estudo restrospectivo [Characterization of the cases referred and consulted in a child and adolescent psychiatric clinic: a retrospective study]. Acta Med Port. 2011;24(6):925-34. Portuguese

5. Meltzer H, Gatward R, Goodman R, Ford T. Mental health of children and adolescents in Great Britain. Int Rev Psychiatry. 2003;15(1-2):185-7.

6. Langsford S, Houghton S, Douglas G, Whiting K. Prevalence and comorbidity of child and adolescent disorders in Western Australian mainstream school students. Psychiatry. 2001;22(1-12).

7. Sawyer MG, Arney FM, Baghurst PA, Clark JJ, Graetz BW, Kosky RJ, et al. The mental health of young people in Australia: key findings from the child and adolescent component of the national survey of mental health and well-being. Aust N Z J Psychiatry. 2001;35(6):806-14.

8. U.S. Department of Health and Human Services. Mental health: a report of the Surgeon General. Rockville, MD, U.S. Department of Health and Human Services, Substance Abuse and Mental Health Services Administration, Center for Mental Health Services, National Institutes of Health. Washington: National Institute of Mental Health; 1999.

9. Santos MI, Coelho I, Rosário F, Machado P, Nery L, Ribeiro J, et al. Referenciação aos cuidados de saúde secundários em idade pediátrica [An analysis of referrals from primary care to a pediatric hospital outpatient clinic]. Rev Port Clin Geral. 2011;27(5):422-32. Portuguese

10. Jordão JG. A medicina geral e familiar: caracterização da prática e sua influência no ensino pré-graduado [Dissertation]. Lisboa: Faculdade de Medicina da Universidade de Lisboa; 1995.

11. Rubio Arribas V, Rodríguez Ibáñez ML, Sampedro Martínez E, Victores Benavente C, Alechiguerra García A, Barrio Gamarra JL. Evaluación de la calidad de comunicación entre niveles asistenciales mediante el documento interconsulta [Evaluation of the quality of communication between care levels through the inter-consultation document]. Aten Primaria. 2000;26(10):681-4. Spanish

12. Morera J, Custodi J, Sánchez L, Miaja F. Análisis de la calidad de la in- formación transmitida entre atención primaria y atención especializada. Medifam. 1995;1(3):132-40.

13. Huertas Zarco I, Pereiró Berenguer J, Sanfélix Genovés J, Rodríguez Moya R. Mejora de la calidad de la hoja de interconsulta a través de la información [Improvement in quality of the interclinical referral note on account of information given]. Aten Primaria. 1996;17(5):317-20. Spanish

14. Irazábal Olabarrieta L, Gutiérrez Ruiz B. Funciona la comunicación entre los niveles primario y secundario? [Does the communication between primary and secondary levels function?] Aten Primaria. 1996;17(6):376-81. Spanish

15. Janeiro M. Acesso aos cuidados de saúde secundários numa extensão do Centro de Saúde de Serpa: 10 anos depois [Access to secondary health care services in a Serpa health centre outpost: tem years on]. Rev Port Clin Geral. 2001;17(3):193-207. Portuguese

16. Anderson MA, Helms LB. Comparison of continuing care communication. Image J Nurs Sch. 1998;30(3):255-60.

17. García Montesinos AM, Odriozola Aranzábal G. Evaluación de la calidad de comunicación entre Atención Primaria y Ginecología de segundo nivel. Centro de Salud. 2001;9(10):628-30.

18. Delgado A, Melquizo M, Guerrero JC, Arboledas A, Revilla L. Análisis de las interconsultas de un centro de salud urbano. Aten Primaria. 1989;5:359-64.

19. Ribeiro C, Marques C. A medicina geral e familiar e a saúde mental infantil: um encontro necessário. Rev Port Clin Geral. 2009;25(5):567-8.

20. Moreno-Martínez F, Casals-Sánchez JL, Sánchez-Rivas JM, Rivera-Irigoin $R$, Vásquez Sánchez MA. Documento de interconsulta: evaluación de la calidad de la comunicación entre atentión primaria y especializada [Referral letter: evaluation of the quality of communication between primary health care and specialist care]. Semergen. 2008;34(5):218-23. Spanish

21. Comin E, Barrio C, Borrel F, Mellorqui C, Esteban C. Información contenida en los volantes de interconsulta en asistencia primaria. Aten Primaria. 1985;2(2):120.

22. Velasco V, Otero A. Evaluación de la calidad de la información médica entre los niveles de atención primaria y especializada. Aten Primaria. 1993;11(8):48-57.

23. Aranaz Andrés JM, Buil Aina JA. Gestión sanitaria: acerca de la coordinación entre niveles asistenciales. Med Clin (Barc) 1986;106:182-4.

24. Ponte CM, Moura BG, Cerejo AC, Braga R, Marques I, Teixeira A, et al. Referenciação aos cuidados de saúde secundários. Rev Port Clin Geral. 2006;22(5):555-68.

25. Barreiro S. Referenciação e comunicação entre cuidados primários e secundários. Rev Port Clin Geral. 2005;21(6):545-53.

26. Sá $A B$, Jordão JG. Estudo europeu sobre referenciação em cuidados primários. II - Dados de seguimento e informação de retorno. Rev Port Clin Geral. 1994;11:25-8.

27. Sá $A B$, Jordão JG. Estudo europeu sobre referenciação em cuidados primários. III - Comparações Internacionais. Rev Port Clin Geral. 1994;11:115-24.

28. WONCA - Comissão Internacional de Classificações da WONCA. ICPC2, International Classification of Primary Care - second edition. Lisboa: ACSS-APMCG; 2011

29. Vilela L, Fernandes G, Dias G, Lima CP, Lopes R, Gesta C. Caracteriza- 
ção de uma consulta de pedopsiquiatria - primeira infância de um hospital central [Characterization of infancy and early childhood outpatient clinic in a central hospital]. Acta Pediatr Port. 2011;42(3):104-7. Portuguese

30. Rushton J, Bruckman D, Kelleher K. Primary care referral of children with psychosocial problems. Arch Pediatr Adolesc Med. 2002;156(6):592-8.

31. Marcelli D, Braconnier A. Infância e psicopatologia. Lisboa: Climepsi; 2005. ISBN 9789728449865

32. Martin A, Volkmar FR. Lewis's child and adolescent psychiatry: a comprehensive textbook. 4th ed. New York: Lippincott Williams \& Wilkins; 2007. ISBN 9780781762144

33. Rutter M, Bishop D, Pine D, Scott S, Stevenson J, Taylor E, et al. Rutter's child and adolescent psychiatry. 5th ed. Washington:Wiley Blackwell; 2008. ISBN 9781405145497

34. Ferreira T. Em defesa da criança: teoria e prática psicanalítica da infância. Lisboa: Assírio \& Alvim; 2002. ISBN 9789723706994

35. Roberts S, Partridge I. Allocation of referrals within a child and adolescent mental health service. BJ Psychiatr Bull. 1998;22(8):487-9.
36. Portaria $n^{\circ} 1.529 / 2008$, de 26 de Dezembro. Diário da República. $1^{\text {a }}$ série(249).

37. World Health Organization. Relatório mundial da saúde - Saúde mental: nova concepção, nova esperança. Lisboa: Direcção-Geral da Saúde; 2002. ISBN 9726750822

38. Heitor MJ, Bento A, Brito I, Cepeda T, Correia H. Rede de referenciação de psiquiatria e saúde mental. Lisboa: Direcção-Geral da Saúde; 2004.

\section{CONFLITOS DE INTERESSE}

Os autores declaram não ter conflito de interesses.

\author{
ENDEREÇO PARA CORRESPONDÊNCIA \\ Marta Araújo \\ Rua D. Soeiro Mouro, 4550-125 Castelo de Paiva \\ E-mail: martaaraujos@gmail.com
}

Recebido em 28-06-2014

Aceite para publicação em 19-12-2014

\section{ABSTRACT \\ REFERRAL FROM PRIMARY CARE TO A CHILD AND ADOLESCENT PSYCHIATRY UNIT IN THE NORTH OF PORTUGAL: A 2-YEAR ANALYSIS}

Objectives: To characterize the referrals from Primary Health Care (PHC) to the child and adolescent psychiatry consultation unit of the Centro Hospitalar de Vila Nova de Gaia/Espinho (CHVNG/E) and to evaluate the communication between these two levels of care.

Study design: Cross-sectional.

Setting: Child and Adolescent Psychiatry Unit of Psychiatry and Mental Health Service of CHVNG/E.

Population: Our sample included all referrals from primary care to the referral unit, using ALERT P1®, in 2010 and 2011.

Methods: Descriptive analyses were performed regarding gender, age, reason for referral, priority of the referral, priority given by the psychiatrist, reason for refusal or return of referral, waiting time, patient attendance, and quality of the referral letter.

Results: Our analysis was based in 980 referrals (61.5\% male). Patients aged 6-10 years were the most commonly referred (41.4\%). The most frequent reason for referral was 'signs and symptoms of child behaviour' (31.9\%). Of all referrals, $16.9 \%$ were considered urgent by the family doctor. Of these, $11.6 \%$ were considered as urgent by the psychiatrist. Of all referrals, $11.3 \%$ were refused or returned, mainly because of insufficient information (36.1\%). About $90 \%$ of the referrals considered as priorities were scheduled within $\leq 60$ days and almost $100 \%$ of the remaining referrals were scheduled within $\leq 150$ days. Of the 853 scheduled first consultations, $10.5 \%$ of the children missed the consultation and did not ask for another appointment. About $20 \%$ of the referral letters were considered of good quality and about $14 \%$ were considered of poor quality.

Conclusions: Our results suggest that communication between family doctors and child and adolescent psychiatrists is effective. Some aspects need improvement, particularly the definition of urgency and the quality of information in the referral letters.

Keywords: Child and Adolescent Psychiatry; Primary Health Care; Referral. 